\title{
Photochemical Chiral Symmetry Breaking in
}

\section{Alanine}

\author{
Matthias Wohlgemuth and Roland Mitrić* \\ Institute for Physical and Theoretical Chemistry, Julius-Maximilians-University Würzburg, \\ Emil-Fischer-Str. 42, 97074 Würzburg, Germany. \\ E-mail: roland.mitric@uni-wuerzburg.de \\ Phone: +49 (0) 931-31-85135.
}

${ }^{*}$ To whom correspondence should be addressed 


\begin{abstract}
We introduce a general theoretical approach for the simulation of photochemical dynamics under the influence of circularly polarized light in order to explore the possibility of generating enantiomeric enrichment through polarized-light-selective photochemistry. The method is applied to the simulation of the photolysis of alanine, a prototype chiral amino acid. We show that a systematic enantiomeric enrichment can be obtained depending on the helicity of the circularly polarized light that induces the excited state photochemistry of alanine. By analyzing the patterns of the photo-induced fragmentation of alanine we find an inducible enantiomeric enrichment up to $1.7 \%$, which is also in good correspondence to the experimental findings. Our method is generally applicable to complex systems and might serve to systematically explore the photochemical origin of homochirality.
\end{abstract}

\title{
Introduction
}

The building blocks of life on Earth, like enzymes and nucleic acids, are composed of homochiral monomers, namely $L$-amino acids and $D$-sugars, and the origin of this symmetrybreaking enantioselectivity of Nature still remains unknown. Since the first investigations of chiral molecules by Pasteur ${ }^{1}$ many hypotheses and speculations on the source of symmetry breaking have been proposed. The observation of enantiomeric enrichment $(e e)$ of left-handed meteoric amino acids ${ }^{2}$ lead to the proposal that extra-terrestrially formed enantiomerically enriched biomolecules were brought to Earth on meteorites and comets and have been subsequently amplified to the present homo-chiral state. The direct observation of chirality in space is not possible through remote detection. Recently, fascinating space missions have been started in order to explore the chemical composition of comets. Launched eleven years ago, the Philae lander of the ESA Rosetta space craft has landed in 2014 on the $67 \mathrm{P} /$ Churyumov-Gerasimonko $(67 \mathrm{P} / \mathrm{C}-\mathrm{G})$ comet. Among other instruments the lander is equipped with a "Cometary Sampling and Composition" (COSAC) instrument which is 
able to identify, separate and quantify organic molecules, including enantiomers expected to be found in cometary ices ${ }^{3}$. After its complicated landing on the comets surface on 12 th November 2014 most of the experiments were finished two days later. The COSAC module has successfully identified several organic molecules ${ }^{4}$. Unfortunately, due to its disadvantageous final position the Philae lander lost its power, such that not all measured data were transferred to Earth. However, the lander awakened on 13th June 2015 for several days and sent a subset of the 8000 measured data sets, which are partly still under investigation ${ }^{5}$.

The optical activity of chiral molecules is their inherent property that arises as a consequence of the specific interaction of the electric and magnetic dipoles with the electric/magnetic components of the polarized light. Thus, several photochemical mechanisms for generating $e e^{6-8}$ were proposed based on the interaction with polarized light that is present in the interstellar space ${ }^{9,10}$. In circularly polarized light $(\mathrm{CPL})$ the electric and the magnetic field, which are perpendicular to each other, rotate about the axis of the direction of field propagation. The helicity of the rotation determines the handedness of the CPL. The different absorption cross section of left $(L-) \mathrm{CPL}$ and right $(R-) \mathrm{CPL}$ by chiral molecules is known as circular dichroism (CD) and can lead to the preferential photochemical fragmentation of one of the enantiomers, and thus the remaining substance becomes enriched in the other form ${ }^{7,11,12}$. This makes the exposition of extraterrestrial organic material to CPL on cosmic objects a promising mechanism for ee.

Although ultrashort laser pulses are not particularly relevant in the astrochemical context, employing such pulses in the laboratory for the detection of CD opens new perspectives for the application of time-resolved spectroscopy and optimal control by pulse shaping to chiral molecules. Time-dependent CD (TDCD) spectroscopy has lead to mechanistic insights into fundamental photochemical processes ${ }^{13,14}$. Due to the weak CD effect, measuring TDCD is an experimentally challenging task. Rhee et al. presented a vibrational TDCD spectroscopy study employing femtosecond laser pulses ${ }^{15}$. Ultrashort laser pulses were also successfully applied to mass spectrometric measurements for the separation of enantiomers ${ }^{16-19}$. 
From the theoretical point of view dynamical CD effects have only been studied in the frame of electron dynamics simulations with fixed nuclei so far ${ }^{20-24}$. For a two-level chiral model system it has been shown that a state population difference induced upon excitation by left- and right CPL can occur. The origin of CD are the magnetic transition dipole moments, which have the same magnitude but an opposite sign for the two enantiomers. However, the magnetic transition dipole moments are two orders of magnitude smaller than their electric counterparts and the selectivity of excitation depends strongly on the orientation of the electric and magnetic dipole moments to the external laser field ${ }^{20,21}$. Extensive studies on TDCD effects of 3-Methylcyclopentanone, employing laser-driven electron dynamics, demonstrated the influence of polarized laser pulses on electronic state populations and ion yields due to multiphoton excitations ${ }^{22-24}$. As yet, all theoretical investigations however neglect the influence of the nuclear motion leading to nonadiabatic coupling between the electronic states. The explicit treatment of the nuclear dynamics is essential for the understanding of the photochemical processes in chiral molecules under the influence of CPL. In this context, a particularly attractive approach which is generally applicable also to complex systems is based on the semi-classical molecular dynamics "on the fly", in which the nuclear motion is described by classical trajectories and the electronic structure is calculated using various quantum chemical methods. For the field-free nonadiabatic dynamics a variety of approaches have been devised, the most widely used being Tully's surface hopping procedure ${ }^{25,26}$. The inclusion of laser pulses into molecular dynamics simulations has been achieved in the framework of our field-induced surface hopping method (FISH) ${ }^{27}$ and successfully applied, e.g. for the simulation of light-induced photodynamics, time-resolved photoelectron spectroscopy (TRPES) ${ }^{28-30}$ or coherent control ${ }^{27,31,32}$, respectively.

Here we focus on the photodynamics of the amino acid alanine (Ala), which has been subject to numerous experimental studies ${ }^{11,12,33-38}$ and of significant interest in theoretical works ${ }^{39-43}$. Our aim is to explore the photochemistry of Ala induced by the interaction with circularly polarized UV light. Ala is the smallest chiral amino acid and thus serves as a 
prototype system for the investigation of $e e$ under interstellar conditions. In order to study the photodynamics using polarized laser pulses, we have extended our FISH approach ${ }^{27,28}$ by the explicit inclusion of the magnetic field, coupled to the molecule via the magnetic transition dipole moments. This methodology is generally applicable to the investigation of the photochemistry of chiral compounds and it includes both electronic and nuclear dynamics at the mixed quantum-classical level.

\section{Method section}

The simulations are performed by the following steps: First, 3000 initial conditions for an ensemble of trajectories have been generated from a $1 \mathrm{~ns}$ long trajectory in the electronic ground-state at constant temperature $(T=50 \mathrm{~K})$. The temperature has been chosen in order to mimic the temperature of objects in the Kuiper belt, which is presumed to be the region of origin for many comets ${ }^{44}$. In order to average over the molecular orientations we have rotated each starting geometry $\mathbf{R}_{i, D}(t=0)=\mathbf{M}_{z}\left(\alpha_{i}\right) \mathbf{M}_{y}\left(\beta_{i}\right) \mathbf{M}_{z}\left(\gamma_{i}\right) \tilde{\mathbf{R}}_{i, D}$ as well as the velocities by the uniformly randomized Euler angles $\left\{0<\alpha_{i}<2 \pi\right\},\left\{0<\beta_{i}<\pi\right\}$ and $\left\{0<\gamma_{i}<2 \pi\right\}$, respectively. The $L$-enantiomer of Ala has been obtained by inversion of the atomic coordinates of $D$-Ala through the center of coordinates, which has been chosen to be the chiral carbon atom.

From these initial conditions, we have launched our semi-classical FISH molecular dynamics running for $1.5 \mathrm{ps}$ in the manifold of the lowest 4 excited states and the ground-state. The Newtonian equations of motion for the nuclei are integrated using the velocity Verlet algorithm ${ }^{45}$ with a time step $\Delta t=0.1 \mathrm{fs}$, where the gradients on the nuclei are obtained from the multi-reference configuration interaction OM2 method ${ }^{46}$. Along each trajectory $\mathbf{R}(t)$ the electronic degrees of freedom are propagated by solving the time-dependent Schrödinger equation in the manifold of adiabatic electronic states coupled by the electric field $\mathbf{E}(t)$, by the magnetic field $\mathbf{B}(t)$ and by the nonadiabatic coupling terms $\mathbf{d}_{i j}$ : 


$$
\begin{aligned}
i \hbar \dot{c}_{i}(t) & =E_{i}(\mathbf{R}(t)) c_{i}(t)-i \hbar \sum_{j}\left[\mathbf{d}_{i j}(\mathbf{R}(t)) \cdot \dot{\mathbf{R}}(t)\right] c_{j}(t) \\
& -\sum_{j}\left[\boldsymbol{\mu}_{i j}(\mathbf{R}(t)) \cdot \mathbf{E}(t)-\mathbf{m}_{i j}(\mathbf{R}(t)) \cdot \mathbf{B}(t)\right] c_{j}(t) .
\end{aligned}
$$

Here, $E_{i}(\mathbf{R}(t))$ represents the adiabatic energy of the $i$-th electronic state, $\boldsymbol{\mu}_{i j}$ and $\mathbf{m}_{i j}$ are the electric and magnetic transition dipole moments, respectively, and $c_{i}(t)$ are the expansion coefficients of the electronic wave function. The equation was numerically integrated using the 4-th order Runge-Kutta method with a step size of $10^{-5}$ fs.

The electronic state in which the trajectory resides is determined by a stochastic surface hopping algorithm, using the hopping probabilities calculated from the change of the quantum electronic state populations $\rho_{i i}=c_{i}^{*} c_{i}$ according to

$$
P_{i \rightarrow j}=\Theta\left(-\dot{\rho}_{i i}\right) \Theta\left(\dot{\rho}_{j j}\right) \frac{-\dot{\rho}_{i i}}{\rho_{i i}} \frac{\rho_{j j}}{\sum_{k} \Theta\left(\dot{\rho}_{k k}\right) \dot{\rho}_{k k}} \Delta \tau
$$

where the $\Theta$ functions are defined to be one for positive arguments and zero otherwise. Notice that in Eq. (1) the shape as well as the polarization of the laser field are not restricted to specific cases. This leads to a variety of possible pulse envelopes and polarizations, including left and right circular or elliptical polarization, allowing us to apply our formalism for many purposes like control of laser-induced processes or the simulation of spectroscopic observables. It should also be noticed that the transition dipole matrix elements $\boldsymbol{\mu}_{i j}$ and $\mathbf{m}_{i j}$ as well as the nonadiabatic coupling term $\mathbf{d}_{i j}$ in Eq. (1) are parametrically dependent on the nuclear trajectory $\mathbf{R}(t)$ and thus are calculated in each nuclear time step.

Throughout this work, we have used CPL where the laser field is propagated in z-direction and has a Gaussian shaped envelope: 


$$
\mathbf{E}(t)=\mathcal{E}_{0} \cdot \exp \left(-\frac{\left(t-t_{c}\right)^{2}}{2 \sigma}\right) \operatorname{Re}\left[\left(\hat{\mathbf{e}}_{\mathrm{x}}+\hat{\mathbf{e}}_{\mathrm{y}} \exp (\mathrm{i} \delta)\right) \exp (-\mathrm{i} \omega \mathrm{t})\right]
$$

where $\delta=\frac{\pi}{2}$ for $L$-CPL, $\delta=-\frac{\pi}{2}$ for $R$-CPL, respectively. The magnetic field is defined perpendicular to the electric field (Eq. (3)):

$$
\mathbf{B}(t)=\frac{1}{c}\left(\hat{\mathbf{e}}_{z} \times \mathbf{E}(t)\right) .
$$

The pulse has been chosen to have a full width at half maximum (FWHM) of $500 \mathrm{fs}$ ( $\sigma \approx 212$ fs) and is centered at $t_{c} \approx 554 \mathrm{fs}$, such that the molecule is allowed to relax in the excited state for about $0.5 \mathrm{ps}$ after the pulse has ended. During the pulse $(0 \leq \mathrm{t} \leq \mathrm{FWTM} \approx 911$ fs, FWTM: full width at tenth of maximum) the energy conservation due to electronic state change is switched off, under the presumption that all energy originating from the laser pulse is absorbed by the molecule. After 911 fs we switch on energy conservation, employing uniform rescaling of the velocities of the nuclei when a state switch occurs according to the energy difference of the electronic states. The field amplitude has been chosen $\mathcal{E}_{0}=2 \sqrt{2}$ $\mathrm{GV} / \mathrm{m}$, in order to yield a reasonable excitation efficiency to the excited state.

Notice, that the inclusion of the coupling of the field to the electric quadruple moment $\mathbf{Q}$, which is in the same order of magnitude as the magnetic dipole coupling, is straightforward by including a third term of the form $\frac{i \omega}{2 c} \mathbf{Q} \cdot \hat{\mathbf{e}}_{z} \cdot \mathbf{E}(t)$ into Eq. (1). However, analytically the the contribution of electric quadrupole coupling vanishes upon rotational averaging ${ }^{47}$, which has been employed in this study.

The chirality on the four bound carbon atoms is determined with the following algorithm: 1) we assigned the priority of each ligand according to the nuclear charges, 2) the ligand with the lowest priority (hydrogen) is aligned to the $z$-axis and the molecule is projected on the $x$-y-plane and 3) the ligand with the second priority (methyl group) is aligned to the $y$-axis and the molecule is projected on to the $x$-axis. Now, if the ligand with the highest priority 
is on the left of the chiral carbon atom on the $x$-axis we have assigned it to $S$-type and, otherwise to the $R$-type.

\section{Results and Discussion}

We explore the photochemistry of Ala under the influence of circularly polarized light employing the extended version of the FISH method.

Ala has 5 energetically close-lying conformers ${ }^{49}$ in the ground electronic state, which are connected by low energy barriers. In order to sample the most abundant conformers, we have run a 1 ns long molecular dynamics (MD) simulation of $D$-Ala in the electronic groundstate using the multi-reference configuration interaction (MR-CI) OM2 method ${ }^{46}$. In order to obtain statistically reliable $e e$ values in the excited state dynamics simulations, one has to run a large number of trajectories. Thus, we sampled 3000 structures and velocities in regular time intervals from the constant temperature MD. These structures served as starting points for the simulation of the photochemistry of Ala under the influence of the CPL.

CD Spectroscopy. In Figure 1 we present the UV/Vis, electronic CD (ECD) and anisotropy spectra of Ala calculated from the sampled geometries. The oscillator strength $f_{i} \propto\left|\boldsymbol{\mu}_{0 i}\right|^{2}$ in the UV/Vis spectrum (Fig. 1a) is directly linked to the extinction coefficient $\epsilon=\epsilon_{L-C P L}+\epsilon_{R-C P L}$ while the rotatory strength $R_{i} \propto\left|\boldsymbol{\mu}_{0 i}\right|\left|\mathbf{m}_{0 i}\right|$ in the ECD spectrum (Fig. 1b) is related to the differential extinction coefficient $\Delta \epsilon=\epsilon_{L-C P L}-\epsilon_{R-C P L}$. These two quantities can be used to define the anisotropy $g=\Delta \epsilon / \epsilon$ (Fig. 1c), which measures the ratio between $\Delta \epsilon$ and $\epsilon$. It has been experimentally shown that the anisotropy allows, together with the extent of the photolysis reaction, $\xi$, for an estimation of the lower limit for the photochemically inducible ees: $e e \geq\left(1-(1-\xi)^{g / 2}\right)^{11,37}$. The anisotropy factor may be approximated by $g_{i} \approx 4 \frac{R_{0 i}}{f_{0 i}}$, presuming the shape of the CD and absorption signal are the same and the permittivity is set to one ${ }^{50}$. As can be seen from Fig. 1b) and c) the ECD and anisotropy spectra differ in their sign for both enantiomers, where the red curves indicate 
the envelope of the $L$-Ala spectra and the blue curve the envelope of the $D$-Ala spectra.

The UV/Vis spectrum (Fig. 1a) shows two well-separated absorption peaks at about 270 $\mathrm{nm}\left(S_{1}\right)$ and $220 \mathrm{~nm}\left(S_{2}\right)$, respectively. While the $S_{1}$ state shows only a weak absorption in the UV/Vis and ECD spectrum (Fig. 1b), the $S_{2}$ state has high oscillator and rotatory strength. In this spirit the $S_{2}$ state would be a good choice as a target state for the laser driven molecular dynamics. However, it bears only a very small intensity in the anisotropy spectrum (Fig. 1c). In order to obtain high population transfer to the excited state as well as high ee values, we have therefore chosen the $S_{3}$ state, located at $190 \mathrm{~nm}$, as the target state, which has a high anisotropy factor compared to the $S_{2}$ state. The $S_{4}$ state is close to the $S_{3}$ state, but has lower oscillator and rotatory strength. For comparison experimental spectra are given in the right panels of Fig. 1 (Fig. 1d-1f). Note, that the calculated spectra in Fig. 1 have been red-shifted by $15 \mathrm{~nm}$. The UV/Vis spectrum (Fig. 1d), obtained from Ala in methanol solution ${ }^{48}$, indicate a weak band at about $250 \mathrm{~nm}$ and a intense band below $210 \mathrm{~nm}$. Thus, the energies are red-shifted by about $20 \mathrm{~nm}$, which can be attributed to the influence of the polar solvent. The experimental ECD spectra of cold Ala films (Fig. 1e, ${ }^{33}$ ) have a weak band at $200 \mathrm{~nm}$ and two stronger bands at 180 and 158 nm, both with opposite sign compared to the first one. The calculated anisotropy spectra (Fig. 1c) and the experimental obtained anisotropy spectra (Fig. 1f, ${ }^{37}$ ) both exhibit a weak band at about $200 \mathrm{~nm}$, an intense band at about $190 \mathrm{~nm}$ and a weaker band at $180 \mathrm{~nm}$, each with opposite signs. It should be noted however, that the experimental values for the ECD and anisotropy spectra were obtained from Ala films, where the zwitterionic form is the predominant isomer ${ }^{33}$, while we investigated the neutral Ala that is the dominant form in the gas phase. The same holds true for the UV/Vis spectrum obtained in methanol, however, to our knowledge no absorption spectra of Ala films have been published so far. Although the comparison of the measured and predicted spectra is not straightforward, theoretical investigations using density functional theory find a blue shift from $274 \mathrm{~nm}$ to $268 \mathrm{~nm}$ for the $S_{1}$ state when switching from the neutral to the zwitterionic form, while the $S_{2}, S_{3}$ and 
$S_{4}$ states are red-shifted by $14 \mathrm{~nm}, 9 \mathrm{~nm}$ and $17 \mathrm{~nm}$, respectively ${ }^{51}$.

Light-induced Dynamics Simulations. Starting from the 3000 randomly oriented initial conditions for $D$ - and $L$-Ala, respectively, we have run our FISH molecular dynamics for $1.5 \mathrm{ps}$ in the manifold of 4 excited states and the ground-state, including the explicit interaction with polarized light. The details of the simulations and the parameters of the laser fields are given in the Methodological section. Two different field polarizations corresponding to $R$-CPL and $L$-CPL have been employed.

After about 200 fs the $S_{3}$ excited state gets populated due to the excitation with the resonant laser pulse. In the course of the dynamics the population is rapidly transferred to lower excited states due to nonradiative relaxation induced by strong nonadiabatic couplings until the first excited state is reached. In the analysis of the trajectories we observe three photochemical processes: a) up to $93 \%$ of the trajectories fragment in the excited state, b) Ala is completely retained with the initial stereogenic configuration and c) the chiral center isomerize to the opposite enantiomer after fragmentation in the excited state and recombination in the electronic ground-state, where the latter one is a subset of a).

A representative trajectory for the photolysis of $L$-Ala illustrating both mechanisms (isomerization and fragmentation) is presented in Fig. 2. L-Ala is photo-excited to the $S_{3}$ state at 360 fs by $L$-CPL, where it quickly relaxes through $S_{2}$ to the first excited state. In the $S_{1}$ state the fragmentation takes place leading to the loss of the COOH group. Subsequently, the crossing of the $S_{1}$ and the $S_{0}$ state is reached at 440 fs leading to the nonradiative deactivation to the ground electronic state. Here, the hydrogen at the previously chiral center swings through the molecular plane and $\mathrm{COOH}$ recombines to the parent molecule, but this time forming $R$-Ala. Since the pulse has not ceased yet, the formed $R$-Ala is photo-excited again at 590 fs and fragments under the loss of $\mathrm{COOH}$ from the first excited state.

In order to investigate the different excitation capabilities of $R$ - and $L$-CPL for both enantiomers, we have computed the electronic state populations $P_{L}$ for $L$-CPL and $P_{R}$ for $R$ CPL, respectively, and defined the time-dependent circular dichroism as $C D(t)=2 \frac{P_{L}-P_{R}}{P_{L}+P_{R}}{ }^{24}$. 
Since the population is rapidly transferred between the excited states, we show the TDCD values for the ground-state, reflecting the population difference of all excited states with respect to the ground-state. The results are presented in Fig. 3 for $L$-Ala (red curve) and D-Ala (blue curve) as well as for racemic Ala (rac-Ala, green curve), which is obtained from the sum of the populations of $L$ - and $D$-Ala. The CD values can be understood such that if $\mathrm{CD}$ is positive $R$-CPL excites the corresponding enantiomer more than $L$-CPL and vice versa. As expected from the ECD spectra (Fig. 1b) the TDCD for $L$ - and $D$-Ala obtained from MD simulations are opposite in their sign. While the CD values are close to zero at the beginning and the center of the laser pulse (shown as gray area in Fig. 3), they steadily decrease for $D$-Ala or increase for $L$-Ala as the pulse intensity decreases until the final population difference is reached with $C D=4.5 \%$ for $L$-Ala and $C D=-8.2 \%$ for $D$-Ala, respectively. The observed behavior is not surprising since at the beginning of the pulse the electronic state population rapidly oscillates between the excited state and the ground-state, while at the end of the pulse a steady state population is reached. As expected, for rac-Ala the TDCD always remains close to zero and finally ends with a value of approximately $-1.5 \%$. This non-vanishing CD for rac-Ala and the not completely stable CD for $L / D$-Ala at the end of the dynamics might be artificial due to convergence failures upon fragmentation, making the evaluation of the CD values numerically unstable. Nevertheless, the simulation clearly shows the different excitation capabilities of $L$ - and $R$-CPL to alanine.

Enantiomeric Enrichment. The direct comparison of TDCD to the experiment is not straightforward since measuring time-dependent electronic state populations is a challenging task, especially for differences no larger than a few percent, and to our knowledge, no such data are available for Ala so far. Instead, most experiments analyzing the ees in amino acids use gas chromatography combined with time of flight mass spectrometry (GC-TOF-MS) or chiral photoelectron spectroscopy $(\mathrm{PES})$, where e.g. Ala is ionized with polarized laser pulses $^{35,36}$. Since Ala is a fragile molecule in gas phase and undergoes photolysis reactions, we have simulated mass spectra for rac-Ala (Fig. 4) by approximating the ionization as an in- 
stantaneous process. This enables us to directly compare the outcome of our photodynamics simulations to experiments on Ala.

For this purpose, we calculated the charge distribution at $\mathrm{t}=1.5 \mathrm{ps}$ in the cationic ground-state and determined the fragments with a proximity criterion. The obtained Mulliken partial charges were summed over atoms in each fragment and the fragment with a total charge close to +1 was used to simulate the mass spectra shown in Fig. 4. Apart from the parent ion $M^{+}(\mathrm{m} / \mathrm{z}=89)$ the abundant masses are the $\mathrm{m} / \mathrm{z}=44$ and $\mathrm{m} / \mathrm{z}=43$ fragments. While the 44 fragment results from the loss of $\mathrm{COOH}$ by the reaction of $\left[\mathrm{CH}_{3} \mathrm{CH}\left(\mathrm{NH}_{2}\right)(\mathrm{COOH})\right]^{+}$ $\rightarrow\left[\mathrm{CH}_{3} \mathrm{CHNH}_{2}\right]^{+}+\mathrm{COOH}$ and is well described in the literature ${ }^{36,52}$, we also see a significant amount of the 43 fragment which comes either from the loss of formic acid or from the loss of $\mathrm{CO}$ and $\mathrm{H}_{2} \mathrm{O}$, where the charge resides on the remaining $\left[\mathrm{CH}_{2} \mathrm{CHNH}_{2}\right]^{+}$fragment. However, the simulations show that formic acid or $\mathrm{H}_{2} \mathrm{O}+\mathrm{CO}$ are always formed after the loss of $\mathrm{COOH}$ by transfer of a second hydrogen from $\left[\mathrm{CH}_{3} \mathrm{CHNH}_{2}\right]^{+}$to $\mathrm{COOH}$ and thus this fragmentation path is a result of the long relaxation time in the excited state. However, in the experiment the charged fragment is rapidly accelerated by the external electric field and the second hydrogen abstraction is unlikely to happen.

While the fragmentation pattern looks quite similar for $L$ - and $R$-CPL (cf. the difference of the mass spectra in the lower panel of Fig. 4), the enantiomeric composition of the remaining Ala molecules as well as of fragments containing intact stereogenic centers is different. As stated above Ala can either undergo complete fragmentation with the loss of the chiral center or other processes in which an asymmetric carbon atom is retained. The chirality of the fragments has been determined and is presented in right panels of Fig. 4, giving rise to the ee values induced by the polarized laser pulses and is calculated according to:

$$
e e_{L}=\frac{N_{L}-N_{D}}{N_{L}+N_{D}}
$$

where $N_{L}$ and $N_{D}$ are the number of preserved stereogenic centers of $L$ - and $D$-Ala, respec- 
tively. In the case of $L$-CPL $209 S$-type and $202 R$-type centers are preserved or formed, giving rise to an $e e_{L}$ of $1.7 \%$. On the other hand for $R$-CPL $191 S$-type and $197 R$-type centers are present at the end of the dynamics simulations inducing an $e e_{L}$ of $-1.5 \%$. These values are lower than the experimentally determined ees, which range up to $(4.49 \pm 0.14) \%$ for $L$-CPL and $(-4.22 \pm 0.21) \%$ for $R$-CPL, depending on the excitation energy, irradiation time and the environmental conditions ${ }^{11,38}$. However, the enantioselectivity of the photolysis reaction strongly depends on the reaction rate, and the higher ees obtained in experiments rely on photolytic yields of $99.97 \%$, achieved with radiation times of several hours ${ }^{11}$, while we can only account for photolysis of up to $93 \%$ due to a much shorter pulse duration. Using the empirically found formula for the lower limit for the inducible $e e^{11,37}$ with an extent of reaction of $\xi=93 \%$ we obtain a lower bound for the inducible $e e_{L}$ of approximately $1.6 \%$ for the calculated anisotropy at $190 \mathrm{~nm}$ (Fig. 1c) and 1.1\% for the measured anisotropy spectrum (Fig. 1f). Note that due to the high extent of the reaction and the small number of trajectories contributing to the presented $e e_{L}$ values, there is still an errorof about $0.1 \%$, presuming a gaussian distribution. As a proof that the effect is mainly due to the interaction with CPL we also present the mass spectrum for the simulations with linear polarized light (LPL) in the supplementary material, where the fragmentation is still the dominant mechanism, although the fragmentation rate is approximately $12 \%$ lower than for CPL. Counting the number of trajectories where the stereogenic center is still present, we find an $e e$ of $0.0 \%$, as it is expected.

Finally, we also want to point out that, in several trajectories, the stereogenic center is inverted in the electronically excited state. This is indicated by the light shaded areas in Fig. 4. In total $7 R \rightarrow S$ isomerizations (5 with $L$-CPL and 2 with $R$-CPL) and $3 S \rightarrow R$ isomerizations ( 1 with $L$-CPL and 2 with $R$-CPL) have taken place. After photolysis in the excited state, Ala relaxes to the electronic ground state, where the recombination to either the same or opposite enantiomer is possible. This opens also a possibility for the hypothesis, that chiral amino acids can be formed under the influence of CPL. However, due to the small 
amount of trajectories undergoing this inversion, no final conclusion can be made.

The analysis of the influence of the pulse parameters (e.g. pulse length, ellipticity and intensity) on the enantiomeric enrichment is still an open, but challenging topic and will be investigated in future work.

\section{Conclusions}

In summary, we have implemented the extension to our FISH method including magnetic field coupling and polarized laser pulses. We have tested and successfully demonstrated our method on the photolysis dynamics of alanine and have shown that circularly polarized light is able to distinguish dynamically on the molecular level between left- and right-handed enantiomers. The photolysis induced by excitation with circularly polarized light leads to non-vanishing enantiomeric enrichment up to $\pm 1.7 \%$ of either of the enantiomers, depending on the helicity of the included laser field. Additionally, we have shown that inversion of the chiral center in the excited state induced by circularly polarized light is in principle possible. This opens a perspective to enantiomeric enrichment due to photochemistry induced by circularly polarized light and has to be investigated further. Altogether, our findings support the hypothesis that homochirality can be induced by circularly polarized light, which may be responsible for enantiomeric enrichment of chiral molecules on extraterrestrial objects prior to their arrival on Earth.

\section{Supporting Information Available}

Movie of a representative trajectory. Mass spectrum and stereogenic composition of rac-Ala irradiated with linear polarized light. This material is available free of charge via the Internet at http://pubs.acs.org/. 


\section{Acknowledgement}

Financial support by the European Research Council (ERC) Consolidator Grant "DYNAMO" (Grant No. 646737) is gratefully acknowledged.

\section{References}

(1) Pasteur, L. Recherches Sur Les Relations Qui Peuvent Exister Entre La Forme Cristalline Et La Composition Chimique, Et Le Sens De La Polarisation Rotatoire. Ann. Chim. Phys. III 1848, 24, 442-459.

(2) Engel, M. Distribution and Enantiomeric Composition of Amino Acids in the Murchison Meteorite. Nature 1982, 296, 837-840.

(3) Gibney, E. Comet Craft Ready to Wake. Nature 2014, 205, 269-270.

(4) Goesmann, F.; Rosenbauer, H.; Bredehöft, J. H.; Cabane, M.; Ehrenfreund, P.; Gautier, T.; Giri, C.; Krüger, H.; Le Roy, L.; MacDermott, A. J. et al. Organic Compounds on Comet 67P/Churyumov-Gerasimenko Revealed by COSAC Mass Spectrometry. Science 2015, 349 .

(5) Fray, N.; Bardyn, A.; Cottin, H.; Altwegg, K.; Baklouti, D.; Briois, C.; Colangeli, L.; Engrand, C.; Fischer, H.; Glasmachers, A. et al. High-Molecular-Weight Organic Matter in the Particles of Comet 67P/Churyumov-Gerasimenko. Nature 2016, published online O־ Sep 2016, DOI: 10.1038/nature19320.

(6) Meinert, C.; de Marcellus, P.; d'Hendecourt, L. L. S.; Nahon, L.; Jones, N. C.; Hoffmann, S. V.; Bredehöft, J. H.; Meierhenrich, U. J. Photochirogenesis: Photochemical Models on the Absolute Asymmetric Formation of Amino Acids in Interstellar Space. Phys. Life Rev. 2011, 8, 307-330.

(7) Evans, A. C.; Meinert, C.; Giri, C.; Goesmann, F.; Meierhenrich, U. J. Chirality, 
Photochemistry and the Detection of Amino Acids in Interstellar Ice Analogues and Comets. Chem. Soc. Rev. 2012, 41, 5447-5458.

(8) Myrgorodska, I.; Meinert, C.; Martins, Z.; d'Hendecourt, L. L. S.; Meierhenrich, U. J. Molecular Chirality in Meteorites and Interstellar Ices, and the Chirality Experiment on Board the ESA Cometary Rosetta Mission. Angew. Chem. Int. Ed. 2015, 54, 14021412.

(9) Bailey, J.; Chrysostomou, A.; Hough, J. H.; Gledhill, T. M.; McCall, A.; Clark, S.; Menard, F.; Tamura, M. Circular Polarization in Starformation Regions: Implications for Biomolecular Homochirality. Science 1998, 281, 672-674.

(10) Fukue, T.; Tamura, M.; Kandori, R.; Kusakabe, N.; Hough, J. H.; Bailey, J.; Whittet, D. C. B.; Lucas, P. W.; Nakajima, Y.; Hashimoto, J. Extended High Circular Polarization in the Orion Massive Star Forming Region: Implications for the Origin of Homochirality in the Solar System. Orig. Life Evol. Biosph. 2010, 40, 335-346.

(11) Meinert, C.; Cassam-Chenai, P.; Jones, N. C.; Nahon, L.; Hoffmann, S. V.; Meierhenrich, U. J. Anisotropy-Guided Enantiomeric Enhancement in Alanine Using Far-UV Circularly Polarized Light. Orig. Life Evol. Biosph. 2015, 45, 149-161.

(12) Modica, P.; Meinert, C.; de Marcellus, P.; Nahon, L.; Meierhenrich, U. J.; le Sergeant d'Hendecourt, L. Enantiomeric Excesses Induced in Amino Acids by Ultraviolet Circularly Polarized Light Irradiation of Extraterrestrial Ice Analogs: a Possible Source of Asymmetry for Prebiotic Chemistry. Astrophysical Journal 2014, 788, 79.

(13) Dartigalongue, T.; Niezborala, C.; Hache, F. Subpicosecond UV Spectroscopy of Carbonmonoxy-Myoglobin: Absorption and Circular Dichroism Studies. Phys. Chem. Chem. Phys. 2007, 9, 1611-1615.

(14) Meyer-Ilse, J.; Akimov, D.; Dietzek, B. Ultrafast Circular Dichroism Study of ohe Ring Opening of 7-Dehydrocholesterol. J. Phys. Chem. Lett. 2012, 3, 182-185. 
(15) Rhee, H.; June, Y.-G.; Lee, J.-S.; Lee, K.; Ha, J.-H.; Kim, Z. H.; Jeon, S.-J.; Cho, M. Femtosecond Characterization of Vibrational Optical Activity of Chiral Molecules. $\mathrm{Na}-$ ture 2009, 458, 310-313.

(16) Boesl, U.; Bornschlegl, A. Circular Dichroism Laser Mass Spectrometry: Differentiation of 3-Methylcyclopentanone Enantiomers. Chem. Phys. Chem. 2006, 7, 2085-2087.

(17) Li, R.; Sullivan, R.; Al-Basheer, W.; Pagoni, R. M.; Compton, R. N. Linear and Nonlinear Circular Dichrosim of R-(+)-3-Methylcyclopentanone. J. Chem. Phys. 2006, 125, 144304.

(18) Bornschlegl, A.; Logé, C.; Boesl, U. Investigation of CD Effects in the Multi Photon Ionisation of R-(+)-3Methylcyclopentanone. Chem. Phys. Lett. 2007, 447, 187.

(19) Breunig, H. G.; Urbasch, G.; Horsch, P.; Cordes, J.; Koert, U.; Weitzel, K.-M. Circular Dichroism in Ion Yields of Femtosecond-laser Mass Spectrometry. Chem. Phys. Chem. 2009, 10, 1199.

(20) Ma, Y.; Salam, A. On Chiral Selectivity of Enantiomers Using a Circular Polarized Pulsed Laser Under Resonant and Off-resonant Conditions. Chem. Phys. 2006, 324, 367.

(21) Ma, Y.; Salam, A. Controlling State Population of Enantiomers of Real Chiral Molecules by Using a Circular Polarized Pulsed Laser. Chem. Phys. Lett. 2006, 431, 247.

(22) Kröner, D. Chiral Distinction by Ultrashort Laser Pulses: Electron Wavepacket Dynamics Incorporating Magnetic Interactions. J. Phys. Chem. A 2011, 115, 14510-14518.

(23) Kröner, D.; Gaebel, T. Circular Dichroism in Mass Spectrometry: Quantum Chemical Investigations for the Differences Between (R)-3-Methylcyclopentanone and Its Cation. J. Phys. Chem. A 2015, 119, 9167-9177. 
(24) Kröner, D. Laser-driven Electron Dynamics for Circular Dichroism in Mass Spectrometry: From Onephoton Excitations to Multiphoton Ionization. Phys. Chem. Chem. Phys. 2015, 17, 19643-19655.

(25) Tully, J. C. Molecular Dynamics with Electronic Transitions. J. Chem. Phys. 1990, 93, 1061.

(26) Hammes-Schiffer, S.; Tully, J. C. Proton Transfer in Solution: Molecular Dynamics with Quantum Transitions. J. Chem. Phys. 1994, 101, 4657.

(27) Mitrić, R.; Petersen, J.; Bonačić-Koutecký, V. Laser-field-induced Surface-Hopping Method for the Simulation and Control of Ultrafast Photodynamics. Phys. Rev. A 2009, 79, 053416.

(28) Mitrić, R.; Petersen, J.; Wohlgemuth, M.; Werner, U.; Bonačić-Koutecký, V.; Wöste, L.; Jortner, J. Time-resolved Femtosecond Photoelectron Spectroscopy by Field-induced Surface Hopping. J. Phys. Chem. A 2011, 115, 3755-3765.

(29) Mitrić, R.; Petersen, J.; Wohlgemuth, M.; Werner, U.; Bonačić-Koutecký, V. Fieldinduced Surface Hopping Method for Probing Transition State Nonadiabatic Dynamics of $\mathrm{Ag}(3)$. Phys. Chem. Chem. Phys. 2011, 13, 8690-8696.

(30) Stanzel, J.; Neeb, M.; Eberhardt, W.; Lisinetskaya, P. G.; Petersen, J.; Mitrić, R. Switching from Molecular to Bulklike Dynamics in Electronic Relaxation of a Small Gold Cluster. Phys. Rev. A 2012, 85, 013201.

(31) Lisinetskaya, P. G.; Mitrić, R. Simulation of Laser-induced Coupled Electron-nuclear Dynamics and Time-resolved Harmonic Spectra in Complex Systems. Phys. Rev. A 2011, 83, 033408 .

(32) Petersen, J.; Wohlgemuth, M.; Sellner, B.; Bonačić-Koutecký, V.; Lischka, H.; 
Mitrić, R. Laser Pulse Trains for Controlling Excited State Dynamics of Adenine in Water. Phys. Chem. Chem. Phys. 2012, 14, 4687-4694.

(33) Meierhenrich, U. J.; Filippi, J.-J.; Meinert, C.; Bredehoeft, J. H.; Takahashi, J.; Nahon, L.; Jones, N. C.; Hoffmann, S. V. Circular Dichroism of Amino Acids in the Vacuum-Ultraviolet Region. Angew. Chem. Int. Ed. 2010, 49, 7799-7802.

(34) Powis, I.; Rennie, E.; Hergenhahn, U.; Kugeler, O.; Bussy-Socrate, R. Investigation of the Gas-phase Amino Acid Alanine by Synchrotron Radiation Photoelectron Spectroscopy. J. Phys. Chem. A 2003, 107, 25-34.

(35) Tia, M.; de Miranda, B. C.; Daly, S.; Gaie-Levrel, F.; Garcia, G. A.; Powis, I.; Nahon, L. Chiral Asymmetry in the Photoionization of Gas-Phase Amino-acid Alanine at Lymanalpha Radiation Wavelength. Phys. Chem. Lett. 2013, 4, 2698-2704.

(36) Tia, M.; de Miranda, B. C.; Daly, S.; Gaie-Levrel, F.; Garcia, G. A.; Nahon, L.; Powis, I. VUV Photodynamics and Chiral Asymmetry in the Photoionization of Gas Phase Alanine Enantiomers. J. Phys. Chem. A 2014, 118, 2765-2779.

(37) Meinert, C.; Bredehoeft, J. H.; Filippi, J.-J.; Baraud, Y.; Nahon, L.; Wien, F.; Jones, N. C.; Hoffmann, S. V.; Meierhenrich, U. J. Anisotropy Spectra of Amino Acids. Angew. Chem. Int. Ed. 2012, 51, 4484-4487.

(38) Meinert, C.; Hoffmann, S. V.; Cassam-Chenai, P.; Evans, A. C.; Giri, C.; Nahon, L.; Meierhenrich, U. J. Photonenergy-Controlled Symmetry Breaking with Circularly Polarized Light. Angew. Chem. Int. Ed. 2014, 53, 210-214.

(39) Powis, I. Photoelectron Circular Dichroism of the Randomly Oriented Chiral Molecules Glyceraldehyde and Lactic Acid. J. Chem. Phys. 2000, 112, 301.

(40) Fukuyama, T.; Matsuo, K.; Gekko, K. Vacuum-Ultraviolet Electronic Circular Dichro- 
ism of L-Alanine in Aqueous Solution Investigated by Time-dependent Density Functional Theory. J. Phys. Chem. A 2005, 109, 6928-6933.

(41) Osted, A.; Kongsted, J.; Mikkelsen, K. V.; Christiansen, O. The Electronic Spectrum of the Micro-Solvated Alanine Zwitterion Calculated Using the Combined Coupled Cluster/Molecular Mechanics Method. Chem. Phys. Lett. 2006, 429, 430-435.

(42) Jaeger, H. M.; III, H. F. S.; Demaison, J.; Csaszar, A. G.; Allen, W. D. LowestLying Conformers of Alanine: Pushing Theory to Ascertain Precise Energetics and Semiexperimental Re Structures. J. Chem. Theory Comput. 2010, 6, 3066-3078.

(43) Farrokhpour, H.; Fathi, F.; Naves De Brito, A. Theoretical and Experimental Study of Valence Photoelectron Spectrum of D,L-Alanine Amino Acid. J. Phys. Chem. A 2012, 116, 7004-7015.

(44) Jewitt, D. C.; Luu, J. Crystalline Water Ice on the Kuiper Belt Object (50000) Quaoar. Nature 2004, 432, 731-733.

(45) Swope, W. C.; Andersen, H. C.; Berens, P. H.; Wilson, K. R. A Computer Simulation Method for the Calculation of Equilibrium Constants for the Formation of Physical Clusters of Molecules: Application to Small Water Clusters. J. Chem. Phys. 1982, 76, 637.

(46) Koslowski, A.; Beck, M. E.; Thiel, W. Implementation of a General Multireference Configuration Interaction Procedure with Analytic Gradients in a Semiempirical Context Using the Graphical Unitary Group Approach. J. Comput. Chem. 2003, 24, 714-726.

(47) Meath, W. J.; Power, E. A. On the Interaction of Elliptically Polarized Light with Molecules; the Effects of Both Permanent and Transition Multipole Moments on Multiphoton Absorption and Chiroptical Effects. J. Mod. Opt. 1989, 36, 977-1002. 
(48) Abu-Eittah, R.; Obaid, A.; Basahl, S.; Diefallah, E. Molecular Orbital Treatment of Some Amino Acids. Bull. Chem. Soc. Jpn. 1988, 61, 2609-2613.

(49) Blanco, S.; Lesarri, A.; Lopez, J.; Alonso, J. L. The Gas-phase Structure of Alanine. J. Am. Chem. Soc. 2004, 126, 11675-11683.

(50) Schellman, J. Circular-dicroism and Optical-Rotation. Chem. Rev. 1975, 75, 323-331.

(51) Kumar, S.; Rai, A. K.; Rai, S.; Rai, D.; Singh, A.; Singh, V. Infrared, Raman and Electronic Spectra of Alanine: a Comparison with Ab Intio Calculation. J. of Mol. Struct. 2006, 791, 23-29.

(52) Jochims, H.-W.; Schwell, M.; Chotin, J.-L.; Clemino, M.; Dulieu, F.; Baumgärtel, H.; Leach, S. Photoion Mass Spectrometry of Five Amino Acids in the 6-22 eV Photon Energy Range. Chem. Phys. 2004, 298, 279-297. 


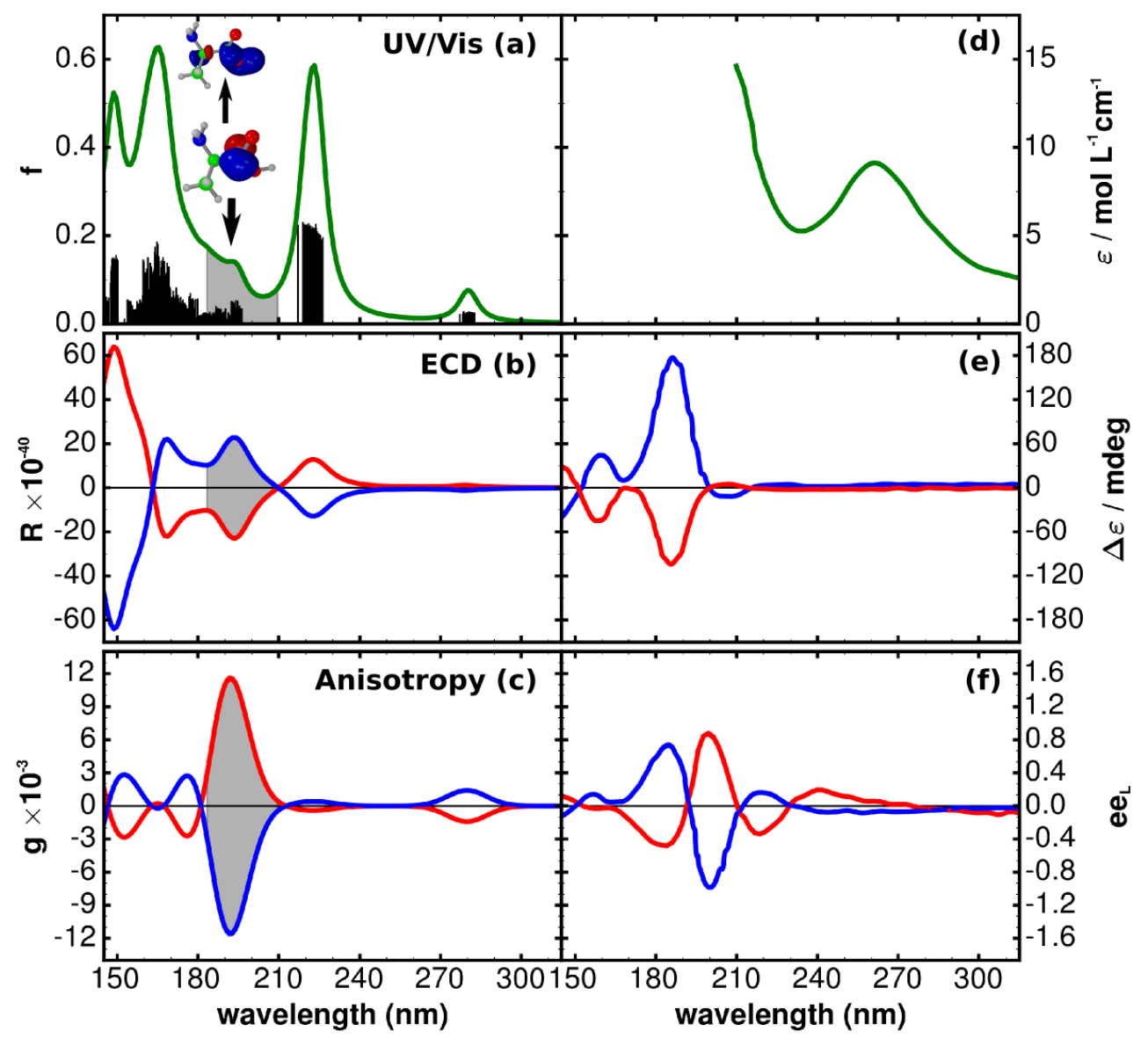

Figure 1: Left panels: (a) UV/Vis, (b) ECD (in cgs units) and (c) anisotropy spectra derived from the 3000 initial structures for the MD simulations. The sticks (shown in (a)) represent the electronic excitation energies and oscillator strength for each geometry in the ensemble of initial conditions. Each stick is convoluted by a Lorentzian with a width of $12 \mathrm{~nm}$. The inset in (a) shows the dominant contribution to the $S_{3}$ electronic excited state, which is of $\pi \rightarrow \pi^{*}$ character. For the ECD (b) and anisotropy (c) spectra the red line indicate the envelope of the spectra $L$-Ala and the blue line the envelope for the $D$-Ala spectra and are given in cgs units. The shaded area indicate the region of highest anisotropy. The spectra have been shifted by $15 \mathrm{~nm}$. Right panels: Experimental (d) UV/Vis spectrum recorded in methanol solution ${ }^{48}$, (e) ECD ${ }^{33}$ and (f) anisotropy spectra obtained from thin films ${ }^{37}$ of $L$ - (red line) and $D$-Ala (blue line). The right scale of the anisotropy spectra gives an estimate for the inducible $e e_{L}$ according $e e \geq\left(1-(1-\xi)^{g / 2}\right)$ using an extent of reaction of $\xi=93 \%{ }^{11,37}$. 

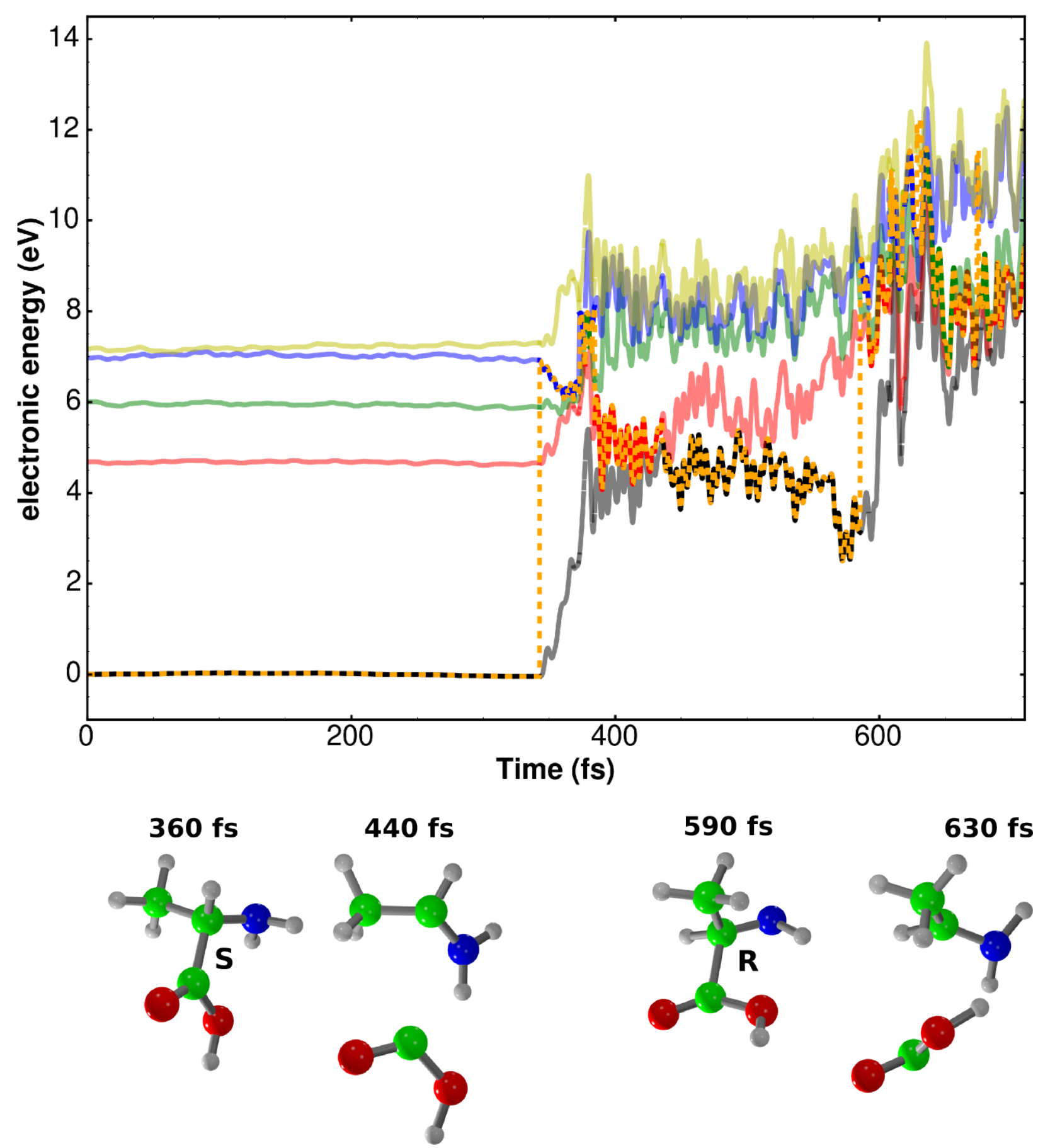

Figure 2: Electronic state energies and snapshots of a representative trajectory of $L$-Ala irradiated with $L$-CPL illustrating the fragmentation as well as the recombination to R-Ala. The black, red, green, blue and yellow lines indicate the energy of the $S_{0}, S_{1}, S_{2}, S_{3}$ and $S_{4}$ electronic state, respectively. The orange dotted line represents the electronic state in which the trajectory resides at a given time. 


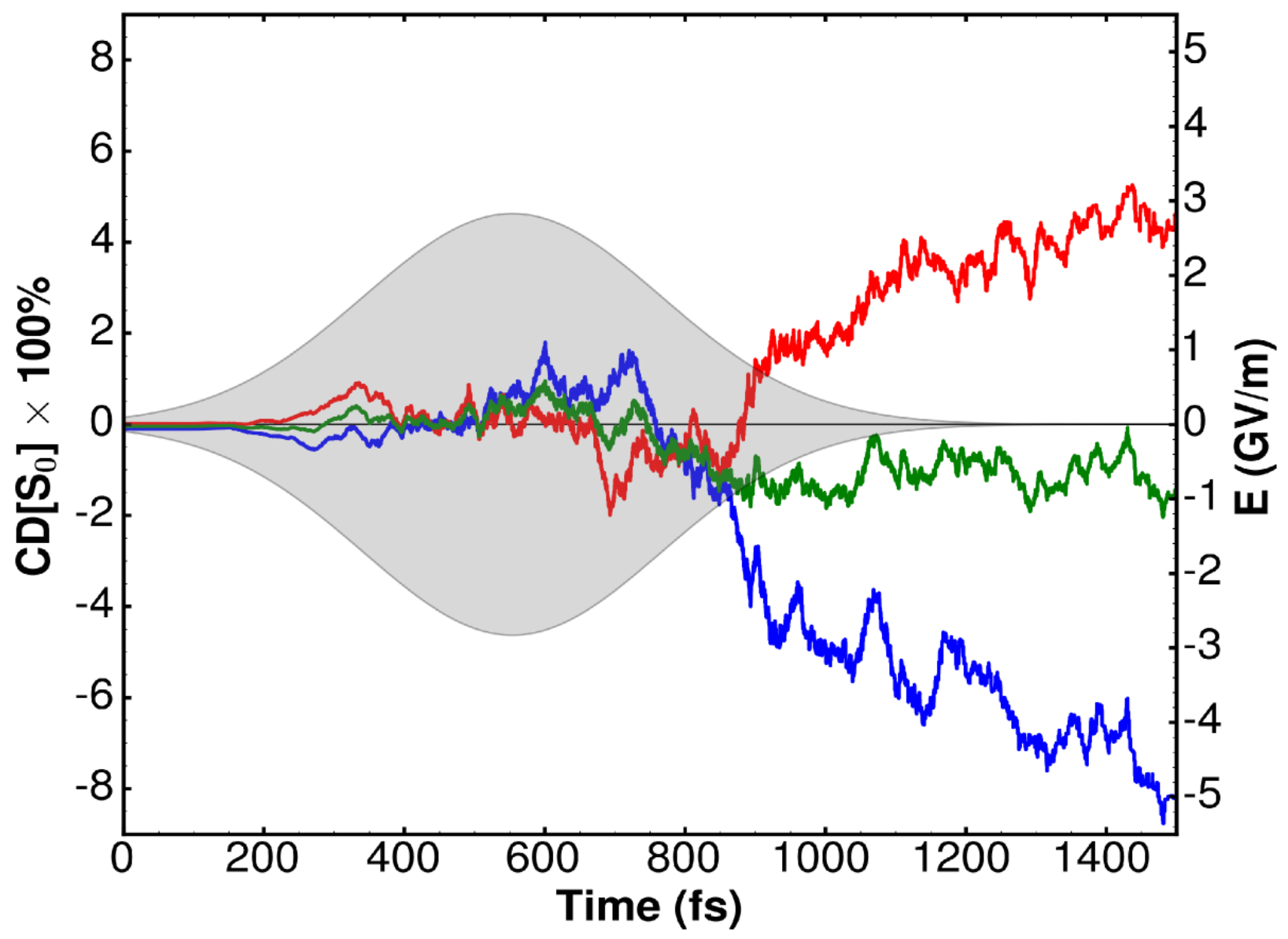

Figure 3: Time-dependent circular dichroism (TDCD) for the electronic ground state for L-Ala (red curve), D-Ala (blue curve) and rac-Ala (green curve). The Gaussian-shaped field envelope $|\mathbf{E}(t)|$ is indicated as gray shaded area. 


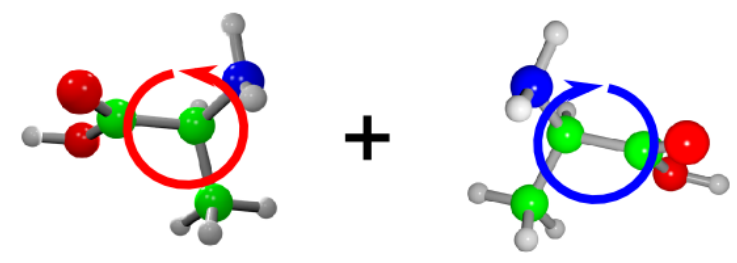

L-Alanine D-Alanine

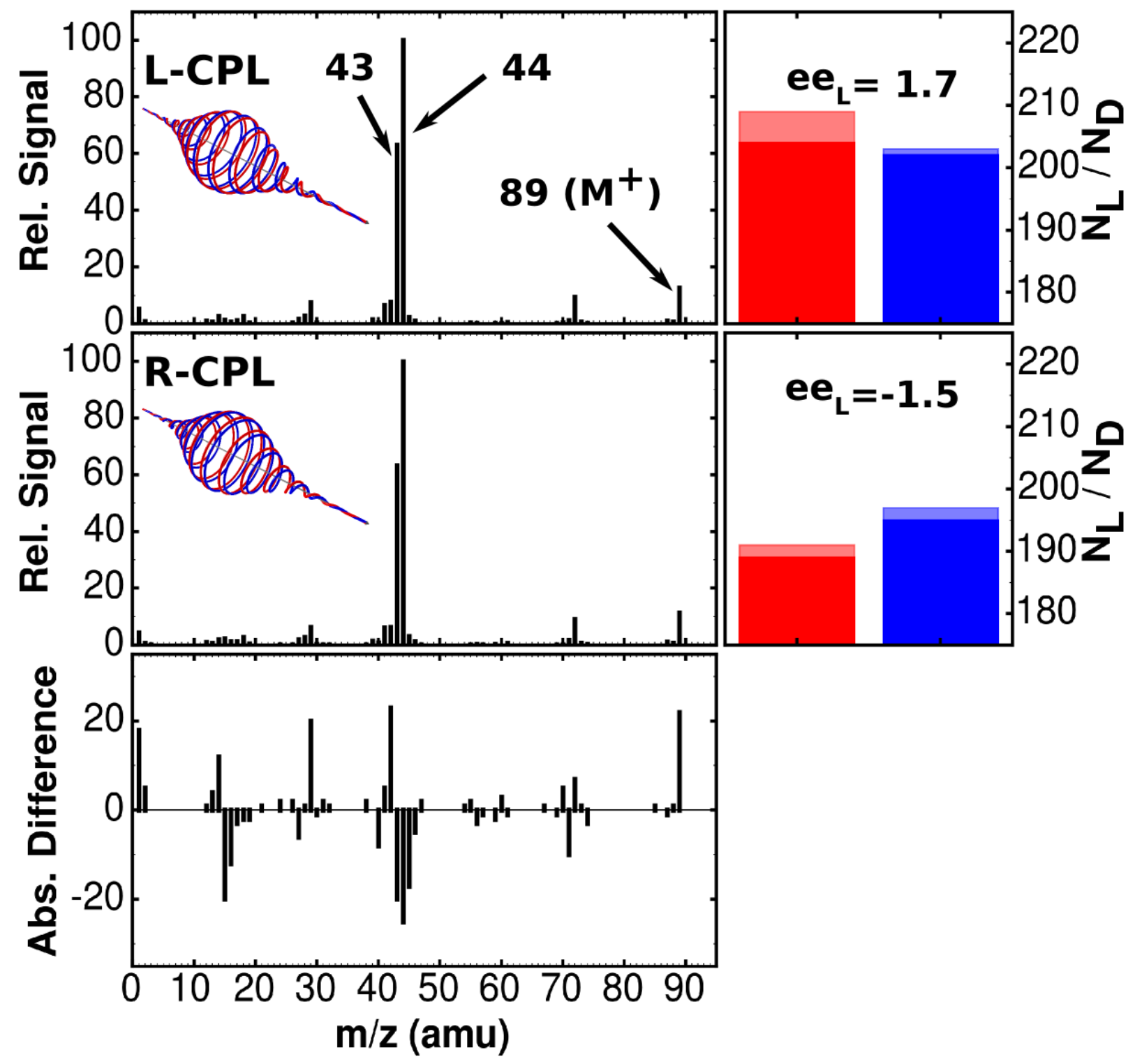

Figure 4: On the left: Mass-spectra of rac-Ala obtained from MD simulations after irradiation with $L$-CPL (upper panel) and $R$-CPL (middle panel), respectively. On the right: Number of trajectories having an $S$ (red) or $R$ (blue) chiral carbon at the end of the dynamics. The lighter areas indicate the number of trajectories where an inversion due to isomerization of the chiral center has taken place. The lower panel shows the difference of both mass spectra in absolute units. 


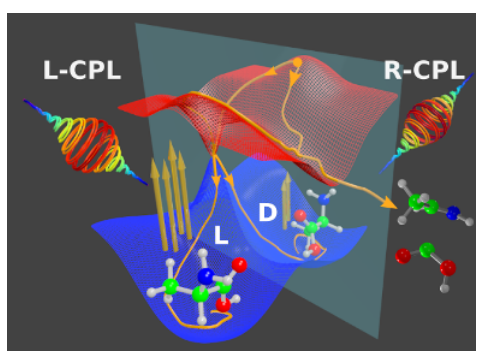

